\title{
Metastatic Prostate Cancer Synchronous with Male Breast Papillary Ductal Carcinoma in situ: Management Dilemma and Literature Review
}

\author{
Harissa Husainy Hasbullaha, b Farah Wahida MdYusofc \\ Amirah Hayati Ahmad ${ }^{c}$ Omar Alzallal ${ }^{d, e}$ Sharifah Emilia T. T Sharif ${ }^{d, e}$ \\ ${ }^{a}$ Clinical Oncologist, Faculty of Medicine, University Teknologi MARA (UiTM), Sungai Buloh,

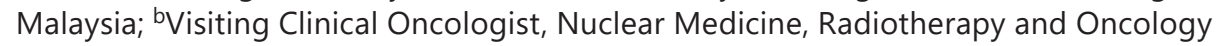 \\ Department, Hospital Universiti Sains Malaysia (HUSM), Kota Bharu, Malaysia; 'Nuclear \\ Medicine, Radiotherapy and Oncology Department, Hospital Universiti Sains Malaysia

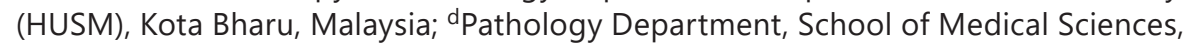

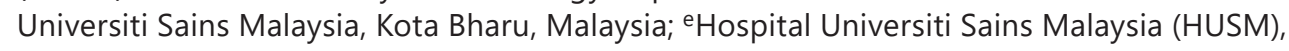 \\ Kota Bharu, Malaysia
}

\section{Keywords}

Ductal carcinoma in situ $\cdot$ Male breast $\cdot$ Prostate cancer $\cdot$ Synchronous

\section{Abstract}

Prostate cancer is common in men, but tumour of the male breast is rare. For these two tumours to be presented synchronously in a male patient is even rarer. The focus of this paper is the case of a 72-year-old man diagnosed with papillary ductal carcinoma in situ after he presented with a unilateral breast mass associated with nipple discharge. Imaging staging for his breast tumour and subsequent prostate biopsy found an incidental synchronous asymptomatic prostate adenocarcinoma as well as bone metastases. He denies risk factors for malignancies and refuses genetic testing. The first part of our discussion will highlight the uncommon occurrence of male breast ductal carcinoma in situ and its management controversies. The subsequent part of our discussion will focus on the association between male breast cancer and prostate cancer, and implication of this on the future treatment of these patients. More importantly, our case will illustrate the challenges in managing dual primaries that present concurrently. on Breast Cancer, October 17, 2019, at Movenpick Hotel, Kuala Lumpur, Malaysia.

\section{Karger"}




\section{Introduction}

Prostate cancer is the second most common cancer among men [1]. Increasing age, black ethnicity, and a family history of malignancies, including those related to BRCA gene mutation, are the established risk factors [2].

However, unlike prostate cancer and female breast carcinoma, male breast cancer is rare, accounting for $<1 \%$ of all breast cancer [3]. The strongest proven risk factors for male breast cancer are a history of breast cancer among first-degree relatives and history of hereditary cancer in the family, especially those that are related to BRCA2 mutation.

Other risk factors for male breast cancer are thought to be due to an increase in the ratio of oestrogen to androgen in the body. These could be due to male patients taking hormonal treatment, especially of oestrogen-based hormones, those with liver disease, obesity, Klinefelter syndrome, and primary testicular disease such as orchitis, cryptorchidism, and testicular trauma $[4,5]$. While a prospective study showed a possible link of smoking to an increased risk of male breast cancer [4], a retrospective analysis on a large US Veteran Affairs database including more than 4 million men disputed this finding [6].

\section{Is Prostate Cancer a Risk Factor for Male Breast Cancer?}

Interestingly, Davis et al. [7] found that while having a history of prostate cancer may be associated with an increased risk of developing a second primary cancer in the urinary bladder, soft tissue including the heart, kidney, and endocrine system, it does not seem to increase the risk of developing a second primary in the male breast any more than that of the general male population. However, in prostate cancer patients harbouring an abnormal gene such as a BRCA2 gene mutation, the risk of developing breast cancer increases significantly [8].

\section{Is Male Breast Cancer a Risk Factor for Prostate Cancer?}

Abhyankar et al. [9] looked at the SEER database of 5,753 male breast cancer cases diagnosed between 1988 and 2012. They found that 250 of these men developed prostate cancer during a median follow-up of 4.3 years. Given that prostate cancer is one of the most common cancers among men, the calculated risk of prostate cancer in patients with a previous history of male breast cancer is no higher than that of the general population.

However, the risk of developing prostate cancer in their lifetime increases 1.9-fold if they also carry a BRCA gene mutation. The risk increases further to 2.64-fold if the mutated gene is BRCA2 [10]. Whether or not there is an association between prostate cancer and male breast cancer, it certainly is not straightforward and will be discussed further in the later part of this report, after our case presentation.

\section{Case Presentation}

A 72-year-old male presented to our centre with a 3-month history of slowly growing, painless right breast swelling associated with bloody nipple discharge. On clinical examination, there was a palpable firm right breast lump $(2 \times 2 \mathrm{~cm})$ in the right retroareolar region with serous discharge from the nipple.

Ultrasound of the right breast showed the presence of a cystic mass with a solid component within the retroareolar region, measuring $1 \times 1 \times 1.8 \mathrm{~cm}$, which was classified as BIRADS 4 . Tissue biopsy of the breast mass demonstrated sheets of mildly pleomorphic tumour cells arranged in a solid papillary architecture with low mitotic activity. p63 immunohistochemistry stain showed positive expression of myoepithelial cells at the periphery of the lesion and

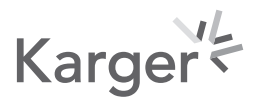




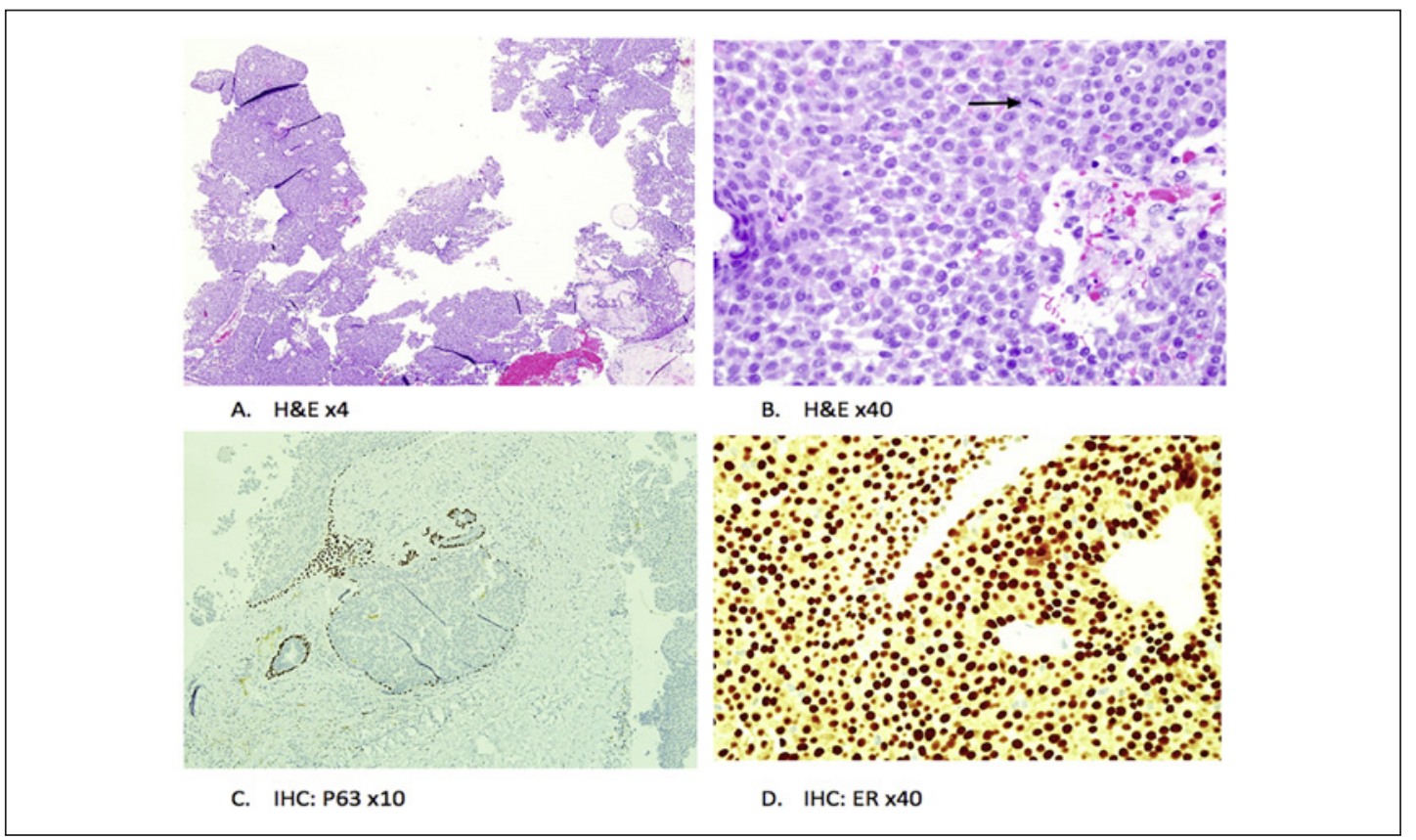

Fig. 1. Histopathology of intraductal papillary carcinoma. Hematoxylin and eosin (H\&E) stains demonstrate sheets of mildly pleomorphic tumour cells arranged in a solid papillary architecture (A) with low mitotic activity (B, arrow). C Immunohistochemistry (IHC) with p63 stain showing positive expression of myoepithelial cells at the periphery of the lesion and absent expression along the papillary fronds. D IHC and ER, showing a positive and diffuse expression.

absent expression along the papillary fronds. A diagnosis of intrapapillary ductal carcinoma in situ (DCIS) was made. The tumour was also diffusely positive for oestrogen receptor (ER) and progesterone receptor (PR), but negative for human epidermal growth factor receptor 2 (HER2). These are demonstrated in Figure 1.

Upon further questioning, the patient denied any risk factors for breast cancer. He has no family history of malignancies or genetic disorders such as Klinefelter syndrome. Apart from hypertension, hyperlipidaemia, and previous cerebrovascular disease from which he completely recovered, he denies any other medical problems, including those which are related to liver disease, thyroid disease, and testicular disease. He is a non-smoker and not particularly overweight.

Subsequently, we proceeded with a computerised tomography (CT) scan for staging purposes. As shown in Figure 2, the scan revealed residual lesion in the right breast and unfortunately also an incidental finding of prostatic mass with bladder infiltration and local regional lymphadenopathy. The CT also showed lytic bony lesions at the L2 vertebrae body, pelvic bone, and left proximal femur. The bone scan shown in Figure 3 confirmed these lesions as metastases. His PSA at diagnosis (iPSA) was $430.7 \mathrm{ng} / \mathrm{mL}$.

A transrectal ultrasound-guided biopsy of the prostatic mass confirmed the diagnosis of prostatic adenocarcinoma with a Gleason score of $8(4+4)$. The patient agreed to mastectomy of the right breast. The histopathology report of the residual breast mass was similar to the biopsy result of papillary DCIS which was ER/PR positive and HER2 negative. There was no microinvasive or invasive component. The surgical margin was clear. We did not perform axillary clearance or a sentinel node biopsy. 
Fig. 2. CT of the pelvis: locally advanced prostate cancer invading the bladder.

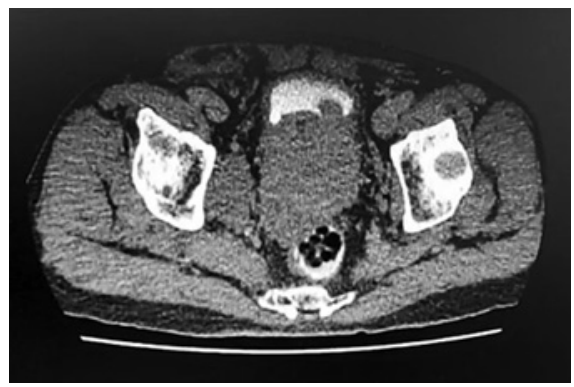

Fig. 3. Bone scan: bone metastases at the L2 vertebrae, left femur, and pelvic bone.

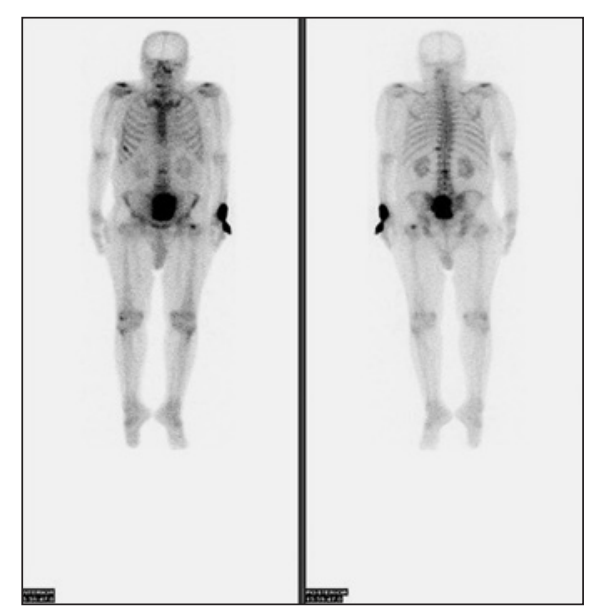

We counselled the patient for genetic testing; however, he refused. The patient was started on anastrazole and subcutaneous leuprolide acetate for treatment of his breast tumour and metastatic prostate cancer. His PSA dropped significantly from 430.7 to $31.3 \mathrm{ng} /$ mL within 3 months after starting leuprolide injection.

\section{Discussion}

\section{Management of Papillary DCIS in Men}

DCIS has been reported to represent only between 9 and 11\% of all male breast cancer as male breast cancers are more commonly invasive in nature $[11,12]$. Because of the rarity of male breast DCIS, its management is mostly extrapolated from that of the management of the female counterpart, with some notable differences in the surgical options as well as endocrine treatment, which will be discussed below.

Papillary DCIS is the least common subtype of female breast DCIS, but it is the most common form of DCIS in male breast cancer [11]. Nevertheless, as with other types of DCIS, untreated papillary DCIS has an increased risk of developing into invasive breast cancer. Therefore, surgical removal of the mass is the mainstay of treatment. For this, we feel that simple mastectomy without axillary surgery was justified as the best approach for our patient.

An American Society of Clinical Oncology (ASCO) expert panel agrees that the surgical option for male breast cancer should be the same as the approach used for women [13]. However, while the option of breast-conserving surgery (BCS) followed by radiotherapy should be discussed with patients, much like with female patients with DCIS, these options are often not practical in the majority of male breast DCIS. This is largely due to the obvious reason of a reduced amount of breast tissue in males compared to females for BCS to achieve 
a safe surgical margin. A large population-based study on male breast cancer reported high a rate of re-excision in patients with in situ disease who did not have a mastectomy. The rate was as high as $60 \%$ [12].

While the no axillary surgery approach in our patient concurs with accepted international guidelines on the management of breast DCIS, such as those from the National Cancer Care Network (NCCN) and ASCO, due to the low rate of axillary metastases associated with DCIS, perhaps we should have considered sentinel lymph node biopsy in the same setting as mastectomy as there is a small possibility that an element of tumour invasiveness may be missed during biopsy. We were fortunate that the final mastectomy specimen of our patient did not reveal an invasive component.

Our case concurs with literature findings that male breast cancer tumours tends to be ER/PR positive and HER2 negative [12]. Evidence for the use of adjuvant endocrine treatment for ER-positive DCIS in female patients is well established [14]. While use of adjuvant tamoxifen in invasive male breast cancer is justified based on the convergence of several retrospective studies on its benefit $[15,16]$, we could not find evidence of adjuvant tamoxifen used for DCIS in male patients.

The decision to embark on adjuvant endocrine treatment in our patient was due to several factors. With the presence of dual primaries, we suspected that our patient has an underlying genetic abnormality that is driving the cancer pathogenesis in him. If this is true, it would put him at higher risk of developing new contralateral breast cancer. For example, BRCA-associated male breast cancer has been reported to have poorer survival compared to its counterparts [17].

Unfortunately, due to the patient's previous history of cerebrovascular accident, he would be at high risk of developing further thromboembolic complications from tamoxifen [18]. The use of aromatase inhibitor alone in male breast cancer has been shown to be less effective than tamoxifen [19]. However, a recently published result from a randomised phase II trial did show that aromatase inhibitor in combination with gonadotrophin-releasing hormone agonist (GnRHa) is more effective at reducing the level of estradiol hormone in male breast cancer patients as opposed to tamoxifen alone [20]. Nevertheless, while the risk of aromatase inhibitor with stroke is less, the use of GnRHa does increase one's risk of stroke [21].

Orchidectomy has been suggested as an alternative to the use of GnRHa in male breast cancer, but evidence is scarce and not enough to support the recommendation, even when it is shown to be less likely to cause stroke compared to GnRHa. The established benefit of GnRHa in effectively treating metastatic prostate cancer as well as its potential role in preventing the recurrence of breast cancer swayed the decision for the use of GnRHa with aromatase inhibitor in our patient. With recent mastectomy, orchidectomy was not considered acceptable by our patient at this moment in time, although it could perhaps be discussed again in the future.

\section{Synchronous Male Breast and Prostate Cancer}

It is most likely that our patient developed cancer in the prostate before the breast, given the advanced stage of prostate cancer as opposed to the early in situ stage of breast tumour at presentation. As we alluded to earlier, having prostate cancer per se does not increase the chance of developing breast cancer [7]. As our patient denies other known risk factors for developing breast cancer, the only factor that was not investigated in him was genetic abnormalities connecting between the two primaries.

It is unlikely that a germline mutation would manifest itself at an age as late as 72 years old, especially in someone with no family history of malignancies. Interestingly, up to $90 \%$ of metastatic prostate cancer patients harbour abnormal genes, either somatic or germline [22].

\section{Karger'}


Even though DNA damage repair (DDR) genes, including the BRCA gene, represent a quarter of abnormal genes in prostate cancer patients, the more common genetic abnormalities detected in them are in the AR, TP53, and RB1 genes. Furthermore, of the DDR genes, while BRCA2 mutation represents the most frequent event (up to $13 \%$ of cases), other abnormal DDR genes have also been detected in metastatic prostate cancer patients, albeit at much lower frequencies, such as the ATM, MSH2, BRCA1, FANCA, MLH1, CHEK, and PTEN genes [22]. These other genes, such as p53, CHEK, and PTEN, also increase the risk for breast cancer [23].

While it is tempting to blame a BRCA2 mutation as the culprit and contributory factor to our patient's dual primary in the breast and prostate, without definitive genetic testing this is only an assumption. It is not impossible that our patient harbours one of the least likely of the possible abnormal genes, or perhaps none at all.

Knowing the exact genetic abnormality in a cancer patient is important in determining the prognosis, and will also have an impact on the surveillance strategy as well as treatment options. For example, castrate-resistant metastatic prostatic cancer patients who progressed after one of the new hormonal treatments would benefit from a targeted drug called olaparib if it is known that they also harbour abnormal DDR genes, especially BRCA1, BRCA2, and ATM [24].

\section{Conclusion}

Our case illustrates the importance of genetic testing in patients with dual primaries, especially so in prostate and male breast cancer as both has been associated with a high risk of harbouring numerous deleterious genetic abnormalities. Even though our patient is currently responding to treatment for his metastatic prostate cancer and his breast tumour is in remission, the exact knowledge of his underlying genetic abnormality would be important in deciding further treatment when the disease progresses. Furthermore, knowing which gene is abnormal would help in surveillance for the manifestation of another possible primary cancer associated with the specific abnormal gene.

Unfortunately for now, the test is not accessible to many as it is non-reimbursable and the price is not affordable to many in our country. Much still needs to be done to increase the uptake of genetic testing among cancer patients.

\section{Acknowledgements}

We thank Dr. Hidayati H. Hasbullah, a trainee Master in Genetics doctor at HUSM Kubang Kerian, for coordinating between clinicians and pathologists.

\section{Statement of Ethics}

The patient gave his written informed consent for the publication of the case and all images used in this article.

\section{Conflict of Interest Statement}

The authors have no conflicts of interest to declare.

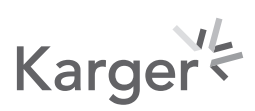




\section{Case Reports in Oncology}

Case Rep Oncol 2021;14:784-791

\begin{tabular}{l|l}
\hline DOI: 10.1159/000515784 & (c) 2021 The Author(s). Published by S. Karger AG, Basel
\end{tabular} www.karger.com/cro

\section{Funding Sources}

There were no sources of funding.

\section{Author Contributions}

F.W.M. and A.H.A. prepared the first draft of the manuscript and prepared the figures. H.H.H. provided the concept, literature review, and critically revised the manuscript. All are involved in the continued care and management of the patient. S.E.T.S. and O.A. are the pathologists responsible for reading the patient's histopathology report and concluding the pathological diagnosis of the tumour. They both also provided input on the pathological perspective of the case. All authors reviewed the final manuscript.

\section{References}

1 Culp MB, Soerjomataram I, Efstathiou JA, Bray F, Jemal A. Recent global patterns in prostate cancer incidence and mortality rates. Eur Urol. 2020 Jan 1;77(1):38-52.

2 Perez-Cornago A, Key TJ, Allen NE, Fensom GK, Bradbury KE, Martin RM, et al. Prospective investigation of risk factors for prostate cancer in the UK Biobank cohort study. Br J Cancer. 2017 Nov;117(10):1562-71.

3 Liu N, Johnson KJ, Ma CX. Male breast cancer: an updated surveillance, epidemiology, and end results data analysis. Clin Breast Cancer. 2018 Oct 1;18(5):e997-1002.

4 Brinton LA, Richesson DA, Gierach GL, Lacey JV Jr, Park Y, Hollenbeck AR, et al. Prospective evaluation of risk factors for male breast cancer. J Natl Cancer Inst. 2008 Oct 15;100(20):1477-81.

5 Giordano SH. Breast cancer in men. N Engl J Med. 2018 Jun 14;378(24):2311-20.

6 Brinton LA, Carreon JD, Gierach GL, McGlynn KA, Gridley G. Etiologic factors for male breast cancer in the US Veterans Affairs medical care system database. Breast Cancer Res Treat. 2010 Jan;119(1):185-92.

7 Davis EJ, Beebe-Dimmer JL, Yee CL, Cooney KA. Risk of second primary tumors in men diagnosed with prostate cancer: a population-based cohort study. Cancer. 2014 Sep 1;120(17):2735-41.

8 Tai YC, Domchek S, Parmigiani G, Chen S. Breast cancer risk among male BRCA1 and BRCA2 mutation carriers. J Natl Cancer Inst. 2007 Dec 5;99(23):1811-4.

9 Abhyankar N, Hoskins KF, Abern MR, Calip GS. Descriptive characteristics of prostate cancer in patients with a history of primary male breast cancer - a SEER analysis. BMC Cancer. 2017 Dec;17(1):659-9.

10 Oh M, Alkhushaym N, Fallatah S, Althagafi A, Aljadeed R, Alsowaida Y, et al. The association of BRCA1 and BRCA2 mutations with prostate cancer risk, frequency, and mortality: a meta-analysis. Prostate. 2019 Jun; 79(8):880-95.

11 Anderson WF, Devesa SS. In situ male breast carcinoma in the Surveillance, Epidemiology, and End Results database of the National Cancer Institute. Cancer. 2005 Oct 15;104(8):1733-41.

12 Harlan LC, Zujewski JA, Goodman MT, Stevens JL. Breast cancer in men in the United States: a populationbased study of diagnosis, treatment, and survival. Cancer. 2010 Aug 1;116(15):3558-68.

13 Hassett MJ, Somerfield MR, Baker ER, Cardoso F, Kansal KJ, Kwait DC, et al. Management of male breast cancer: ASCO guideline. J Clin Oncol. 2020 Jun 1;38(16):1849-63.

14 Staley H, McCallum I, Bruce J. Postoperative tamoxifen for ductal carcinoma in situ. Cochrane Database Syst Rev. 2012;10(10):CD007847.

15 Fentiman IS, Fourquet A, Hortobagyi GN. Male breast cancer. Lancet. 2006 Feb 18;367(9510):595-604.

16 Eggemann H, Brucker C, Schrauder M, Thill M, Flock F, Reinisch M, et al. Survival benefit of tamoxifen in male breast cancer: prospective cohort analysis. Br J Cancer. 2020 May 5;123(1):33-7.

17 Gargiulo P, Pensabene M, Milano M, Arpino G, Giuliano M, Forestieri V, et al. Long-term survival and BRCA status in male breast cancer: a retrospective single-center analysis. BMC Cancer. 2016 Dec;16(1):375-1.

18 Eggemann H, Bernreiter AL, Reinisch M, Loibl S, Taran FA, Costa SD, et al. Tamoxifen treatment for male breast cancer and risk of thromboembolism: prospective cohort analysis. Br J Cancer. 2019 Feb;120(3):301-5.

19 Eggemann H, Ignatov A, Smith BJ, Altmann U, von Minckwitz G, Röhl FW, et al. Adjuvant therapy with tamoxifen compared to aromatase inhibitors for 257 male breast cancer patients. Breast Cancer Res Treat. 2013 Jan; 137(2):465-70.

20 Reinisch M, Seiler S, Hauzenberger T, Kamischke A, Schmatloch S, Strittmatter HJ, et al. Efficacy of endocrine therapy for the treatment of breast cancer in men: results from the MALE phase 2 randomized clinical trial. JAMA Oncol. 2021 Feb 4; 2207442.

21 Jespersen CG, Nørgaard M, Borre M. Androgen-deprivation therapy in treatment of prostate cancer and risk of myocardial infarction and stroke: a nationwide Danish population-based cohort study. Eur Urol. 2014 Apr 1;65(4):704-9. 
22 Messina C, Cattrini C, Soldato D, Vallome G, Caffo O, Castro E, et al. BRCA mutations in prostate cancer: prognostic and predictive implications. J Oncol. 2020 Sep 7;2020:4986365.

23 Economopoulou P, Dimitriadis G, Psyrri A. Beyond BRCA: new hereditary breast cancer susceptibility genes. Cancer Treat Rev. 2015 Jan 1;41(1):1-8.

24 de Bono J, Mateo J, Fizazi K, Saad F, Shore N, Sandhu S, et al. Olaparib for metastatic castration-resistant prostate cancer. N Engl J Med. 2020 May 28;382(22):2091-102. 